\title{
Protocol
}

\section{Perturbation of interactome through micro-RNA and methylome analysis in diabetes endophenotypes: the PIRAMIDE pathogenic clinical study design}

\author{
Giuditta Benincasa $^{1 *}$, Raffaele Marfella ${ }^{1}$, Concetta Schiano ${ }^{2}$, Claudio Napoli ${ }^{1,2}$ \\ ${ }^{1}$ Department of Advanced Clinical and Surgical Sciences, University of Campania "Luigi Vanvitelli, Naples, Italy \\ ${ }^{2}$ IRCCS SDN, Naples, Italy
}

Received: 20 March 2019

Accepted: 23 April 2019

\section{*Correspondence:}

Dr. Giuditta Benincasa,

E-mail: dr.benincasa.giuditta@gmail.com

Copyright: $\odot$ the author(s), publisher and licensee Medip Academy. This is an open-access article distributed under the terms of the Creative Commons Attribution Non-Commercial License, which permits unrestricted non-commercial use, distribution, and reproduction in any medium, provided the original work is properly cited.

\section{ABSTRACT}

Background: The main challenge in type 2 diabetes (T2D) is to detect the regulators of pathogenic events during early stages of disease, as well as prevention and progression follow-up of cardiovascular (CV) complications. DNA methylation and micro-RNAs (miRNAs) are major components of the epigenome, which are involved in the diabetic interactome. This study protocol may contribute to advance our knowledge on molecular basis underlying T2D and its $\mathrm{CV}$ complications, as well as provide putative useful prognostic biomarkers.

Methods: The perturbation of interactome through micro-RNA and methylome analysis in diabetes endophenotypes: the PIRAMIDE pathogenic clinical study protocol is a cross-sectional research program planned to combine big data and network-based analysis aimed to investigate whether DNA methylation and miRNAs may act as simultaneous regulators of the interactome in T2D patients. Clinical datasets will be aggregate to large-scale DNA methylation, mRNA-Seq, and miRNA-Seq analysis performed both on purified $\mathrm{CD} 4^{+}$and $\mathrm{CD} 8^{+} \mathrm{T}$ cells isolated from $35 \mathrm{~T} 2 \mathrm{D}$ patients and 35 sex and age-matched controls. DNA methylome data will be used as input for the weighted human DNA methylation PPI network (WMPN) algorithm. RNA sequencing data will be used as input data for the TargetScan algorithm. The primary endpoint will be to integrate both DNA methylation and miRNA networks to potentially capture which genes are simultaneously modulate by interactions between epigenetic changes. Then, statistical analysis will be performed to correlate these molecular modifications with development of T2D-related CV complications.

Conclusions: PIRAMIDE pathogenic clinical study protocol will test the hypothesis that simultaneous interactions between DNA methylation and miRNAs may hit T2D-associated candidate genes and predict the development of T2D-related CV complications.

Trial Registration: The ongoing PIRAMIDE pathogenic clinical study protocol has been registered on NIH website (NCT03792607).

Keywords: Type 2 diabetes, Prognostic biomarkers, Cardiovascular complications, Network analysis

\section{INTRODUCTION}

Diabetes is a high heterogeneous, metabolic disorder grouped in multifactorial and monogenic forms. ${ }^{1}$ Despite different etiology, hyperglycemic patients are at high risk for developing cardiovascular (CV) complications projected to exceed 23 million deaths annually by 2030 . $^{2-}$ ${ }_{5}^{5}$ Diabetic patients show a strong phenotypic variability concerning clinical presentation at time of diagnosis, as well as type and rate of $\mathrm{CV}$ complications. This evidence leads to frequent difficulties to obtain an early classification and a finer risk stratification of 
population. ${ }^{1,2}$ One explanation arises from the not totally knowledge regarding when, how, and where specific molecular mechanisms are deregulated, thus disturbing the intricate molecular signaling that governs the cellular interactome. Clinically, it means that current therapeutic strategies employ drugs based either on partial or inexact targets. An additional pitfall concerns the lack of effective biomarkers able to early predict T2D patients at high risk of $\mathrm{CV}$ complications, thereby the classical glycemia assessment and dosages of glycosylated hemoglobin (HbA1c), fructosamine, and glycated albumin still show some limitations. ${ }^{6}$ However, innovative randomized trial designs will be crucial to ameliorate evidence-based guidelines leading to a better patient and healthcare outcomes. ${ }^{7-10}$

Despite several genome wide association studies (GWASs) identified some alleles associated with T2Dhigh risk, the most (>95\%) of hyperglycemic patients was unrelated to genetic background highlighting that environmental exposure, such as Western food or sedentary behavior, may play a pivotal role in disease onset. $^{11-14}$ Epigenetic changes can bridge genome to environment, in order to maintain cellular homeostasis without changing DNA sequence. ${ }^{15,16}$ The main classes of epigenetic regulators are DNA and mRNA methylation, histone modifications, as well as noncoding RNAs. ${ }^{15,16}$ Advances in omics technologies have unveiled many epigenetic changes able to alter the expression of gene networks related to T2D onset and its $\mathrm{CV}$ traits, as described in the metabolic memory hypothesis. ${ }^{17-19}$ Interestingly, several investigations reported that aberrant epigenetic changes may be transmitted through meiotic cell divisions (transgenerational effect), thus increasing susceptibility to T2D and atherosclerosis in the offsprings; however, this issue deserves further clinical investigations. ${ }^{20-22}$ Discovering novel epigenetic factors and their interactions represents a promising avenue to ameliorate diagnosis, risk assessment, and personalized approach to treatment of T2D patients. ${ }^{23}$ Currently, network medicine is the most innovative, and potent approach that may identify the molecular dissimilarities that often exist among patients with common clinical phenotype. $^{24-26}$ The application of network-based tools has largely contributed to improve our knowledge regarding both genetic and epigenetic mechanisms underlying T2D-related endophenotypes and its macrovascular complications. ${ }^{27-29}$

The perturbation of interactome through microRNA and methylome analysis in diabetes endophenotypes: the PIRAMIDE pathogenic clinical study design (NCT03792607) is a cross-sectional clinical research network-based approach aimed to identify putative interactions between DNA methylation and microRNAs (miRNAs) associated with T2D-related candidate genes (Figure 1). The second endpoint will be to evaluate the predictive value of these putative epigenetic interactions regarding the insurgence of macrovascular complications in T2D patients respect with controls. This strategy might shed new lights in our understanding of T2D pathogenesis, as well as unveil novel prognostic biomarkers able to improve T2D personalized therapies.

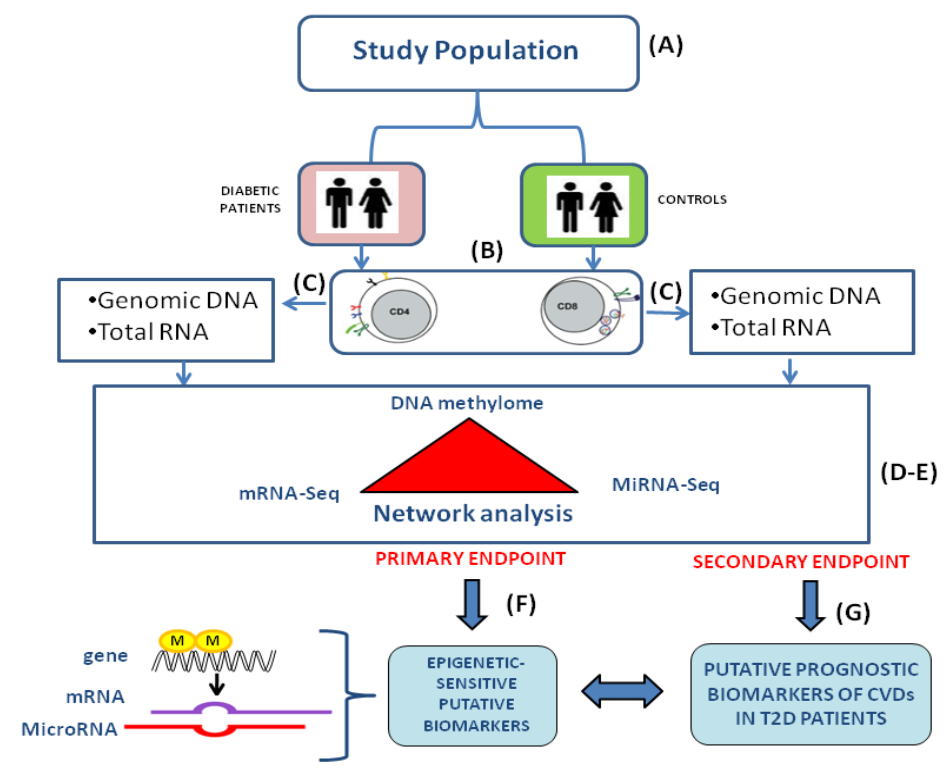

Figure 1: Phases of PIRAMIDE clinical study design. A) Study population encompasses 35 T2D vs 35 controls; B) purification both of CD4+ and CD8+T cells from peripheral blood samples of cases and controls; C) extraction of genomic DNA and total RNA; D) global DNA methylation analysis and RNA-seq; E) Bioinformatics analysis to constuct the interactome; F) Primary end-point: identification of putative interaction between DNA methylation and micro-RNAs; G) Secondary end-point: statistical analysis to validate the predictive power of these putative epigenetic interactions.

CVDs: cardiovascular diseases; T2D: type 2 diabetes; WMPN: weighted human DNA methylation PPI network. 


\section{METHODS}

\section{Study objectives}

The primary objective of the PIRAMIDE pathogenic clinical study design (NCT03792607) is to create an integrative network to identify putative epigenetic interactions between DNA methylation and miRNAs that may synergistically modulate critical T2D-related genes, thus perturbing the interactome flow both of $\mathrm{CD}^{+}$and $\mathrm{CD}^{+}$cells. $^{30}$ The secondary objective will be to correlate these potential candidate genes with the development of macrovascular complications as potential prognostic biomarkers in $\mathrm{T} 2 \mathrm{D}$.

\section{Study population and patient selection}

Population study will be recruited at the Clinical Department of Internal Medicine and Specialistics, Department of Advanced Clinical and Surgical Sciences belonging to University of Campania "Luigi Vanvitelli". This study will be performed according to the principles outlined in the Helsinki Declaration, and has been approved from the local Ethics Committee (Protocol N. 34118). We will enroll 35 men and women 50 years or older with diagnosis of T2D and 35 sex and age-matched controls. In according to current guidelines, diabetes will be diagnosed by evidence of impairing fast glucose (IFG) $\geq 7.0 \mathrm{mmol} / \mathrm{l} \geq 126 \mathrm{mg} / \mathrm{dl}$ ), post prandial glycemia $\geq 11.1$ $\mathrm{mmol} / \mathrm{l}(\geq 200 \mathrm{mg} / \mathrm{dl})$, and evidence of glycated hemoglobin $(\mathrm{HbA} 1 \mathrm{c}) \geq 6.5 \%$. ${ }^{1}$ Clinical and demographic characteristics of the study population will be available from datasets generated by physicians. Patients with known history of cancer, malignancy disorders, active infections, and chronic or immune-mediated diseases will be excluded from the study.

\section{Inclusion criteria}

Inclusion criteria were meets the current American Diabetes Association guideline for diagnosis of diabetes (Diabetic group); must be willing and able to comply with study requirements; must indicate their understanding of the study and willingness to participate by signing an appropriate informed consent form

\section{Exclusion criteria}

Exclusion criteria were history of cancer; malignancy disorders; active infections; chronic or immune-mediated diseases; primary disease requiring surgical intervention; unable to comply with the complication screening; less than $18 \mathrm{y}$ of age; pregnant or are planning to become pregnant during the duration of the investigation; life expectancy $<12 \mathrm{~m}$; currently participating in any other clinical investigation.

Subjects are considered enrolled in the study after giving informed consent; sites then assess the subject inclusion criteria and other clinical and demographic variables at a baseline visit, as reported in Table 1. Diabetes macrovascular complications will be evaluated according to Standards of Medical Care in Diabetes-2018. ${ }^{1}$

Table 1: Summary of all scheduled evaluations and procedures.

\begin{tabular}{|lll|}
\hline \multicolumn{1}{|l|}{ Enrollment } & Baseline & Patient classification \\
\hline Informed consent procedure & $\mathrm{X}$ & \\
\hline Inclusion/exclusion criteria check & $\mathrm{X}$ & $\mathrm{X}$ \\
\hline Diabetes assessment & & \\
\hline Patient data and medical history & $\mathrm{X}$ & \\
\hline Current medications & $\mathrm{X}$ & \\
\hline Changes in medications & $\mathrm{X}$ & $\mathrm{X}$ \\
\hline Patient global assessment & & $\mathrm{X}$ \\
\hline Routine blood test & $\mathrm{X}$ \\
\hline 12-Lead ECG & & $\mathrm{X}$ \\
\hline 6-min hall walking test & $\mathrm{X}$ \\
\hline Echocardiography & & $\mathrm{X}$ \\
\hline Echodoppler TSA & & $\mathrm{X}$ \\
\hline Echodoppler arterial & & \\
\hline Stress ECG test & & \\
\hline
\end{tabular}

\section{Cell separation, nucleic acid extraction, and sequencing analysis}

We will perform the purification both of $\mathrm{CD}^{+}$and $\mathrm{CD}^{+} \mathrm{T}$ cells from peripheral blood samples of cases and controls by using EasySep ${ }^{\mathrm{TM}}$ Human Naïve CD4 ${ }^{+} \mathrm{T}$ Cell Enrichment Kit (Stem Cell) and EasySep ${ }^{\mathrm{TM}}$ Human Naïve $\mathrm{CD}^{+} \mathrm{T}$ Cell Enrichment Kit (Stem Cell), respectively. Then, genomic DNA will be extracted from each cell type by using DNeasy Blood \& Tissue kit (QIAGEN), according to manufacturer protocol. Pooled DNA samples consisting of equal quantities of DNA (2 $\mu \mathrm{g}$ ) from cases and controls will be shipped to Genomix4Life Genomics and Bioinformatics Service, to perform a global DNA methylation analysis. For this aim, it will be used the human methylation $27 \mathrm{~K}$ BeadChip platform by using bisulfite conversion technology. 
Total RNA will be extracted both from $\mathrm{CD} 4^{+}$and $\mathrm{CD} 8^{+}$ cells by using RNeasy Mini Kit (QIAGEN) according to manufacturer protocol. The cDNA library preparation will be performed starting from 4 ug of total RNA by using Illumina TruSeq Libraries and then sequenced at high coverage on the Illumina HiSeq 2500 next generation sequencing (NGS) platform. Nucleic acid quality control from Genomix4Life will be assessed by using Nanodrop spectrophotometer (Thermo Fisher Scientific) and Qubit assay (Thermo Fisher Scientific) and TapeStation 4200 (Agilent).

\section{Bioinformatics analysis}

The weighted human DNA methylation PPI network (WMPN) will be construct to obtain a T2D-related subnetwork based on differentially methylated genes both in $\mathrm{CD} 4+$ and $\mathrm{CD} 8+\mathrm{T}$ cells isolated from cases and controls. $^{28}$

The TargetScan algorithm, by searching the conserved seed pairing regions in the 3' untraslated regions (UTR) of genes based on whole genome alignment, will be used to robustly predict miRNA-target gene pairs both in CD4+ and CD8+ $\mathrm{T}$ cells isolated from cases and controls. $^{31}$

\section{Statistical analysis}

Both univariate and multivariate regression models, as well as receiver operating characteristic (ROC) curve analysis will be performed to validate the predictive power of these putative epigenetic interactions regarding the progression of cardiovascular events in T2D patients.

\section{Ethics approval and consent to participate}

This protocol study will be performed in according to the principles outlined in the Helsinki Declaration and a written informed consent will be obtained from all subjects enrolled. The study was approved from Ethics Committee belonging to University of Campania "Luigi Vanvitelli" (Protocol N. 34118).

\section{CONCLUSION}

Determining the interactome-related molecular alterations in diabetic patients is a challenging task for physicians. The identification of epigenetic-sensitive prognostic biomarkers may offer amplified opportunities to personalize the management of diabetic patients. The PIRAMIDE pathogenic clinical study design will offer a useful network-based research approach to identify putative DNA methylation-miRNA-mRNA axis that might play a critical role in the clinical course of T2D. Furthermore, the second endpoint will be to unveil potential disease-associated hub modules that might be translated in prognostic biomarkers able to predict the clinical progression of T2D macrovascular complications.
Funding: This work was supported by "Ricerca Corrente 2013-2015” (project code RRC-2015-2360454) from Italian Ministry of Health (PI Prof. Napoli). Dr. Giuditta Benincasa is a PhD student of Translational Medicine and she is supported by Educational Grant from the University of Campania, Naples, Italy

Conflict of interest: None declared

Ethical approval: The study was approved by the Institutional Ethics Committee belonging to University of Campania "Luigi Vanvitelli"

\section{REFERENCES}

1. American Diabetes Association. Cardiovascular disease and risk management: standards of medical care in diabetes-2018. Diabetes Care. 2018;41:S86S104.

2. Low Wang CC, Hess CN, Hiatt WR, Goldfine AB. Clinical update: cardiovascular disease in diabetes mellitus: atherosclerotic cardiovascular disease and heart failure in type 2 diabetes mellitus mechanisms, management, and clinical considerations. Circulation. 2016;133:2459-62.

3. Sachdev M, Sun JL, Tsiatis AA, Nelson CL, Mark DB, Jollis JG. The prognostic importance of comorbidity for mortality in patients with stable coronary artery disease. J Am Coll Cardiol. 2004;43:576-82.

4. Global Burden of Metabolic Risk Factors for Chronic Diseases Collaboration. Cardiovascular disease, chronic kidney disease, and diabetes mortality burden of cardiometabolic risk factors from 1980 to 2010: a comparative risk assessment. Lancet Diabetes Endocrinol 2014;2:634-47.

5. Laslett LJ, Alagona P Jr, Clark BA 3rd, Drozda JP Jr, Saldivar F, Wilson SR, et al. The worldwide environment of cardiovascular disease: prevalence, diagnosis, therapy, and policy issues: a report from the American College of Cardiology. J Am Coll Cardiol. 2012;60:1-49.

6. Selvin E, Rawlings AM, Lutsey PL, Maruthur N, Pankow JS, Steffes M, et al. Fructosamine and glycated albumin and the risk of cardiovascular outcomes and death. Circulation. 2015;132:269-77.

7. Pradhan AD, Paynter NP, Everett BM, Glynn RJ, Amarenco P, Elam M, et al. Rationale and design of the pemafibrate to reduce cardiovascular outcomes by reducing triglycerides in patients with diabetes (PROMINENT) study. Am Heart J. 2018;206:8093.

8. Lambrakis K, French JK, Scott IA, Briffa T, Brieger $\mathrm{D}$, Farkouh ME, et al. The appropriateness of coronary investigation in myocardial injury and type 2 myocardial infarction (ACT-2): a randomized trial design. Am Heart J. 2018;208:11-20.

9. Dorcely B, Katz K, Jagannathan R, Chiang SS, Oluwadare B, Goldberg IJ, Bergman M. Novel biomarkers for prediabetes, diabetes, and associated complications. Diabetes Metab Syndr Obes. 2017;10:345-61. 
10. Basu S, Sussman JB, Berkowitz SA, Hayward RA, Yudkin JS. Development and validation of risk equations for complications of type 2 diabetes (RECODe) using individual participant data from randomised trials. Lancet Diabetes Endocrinol. 2017;5:788-798.

11. Sladek R, Rocheleau G, Rung J, Dina C, Shen L, Serre D, et al. A genome-wide association study identifies novel risk loci for type 2 diabetes. Nature. 2007;445:881-5.

12. Scott LJ, Mohlke KL, Bonnycastle LL, Willer CJ, Li Y, Duren WL, Erdos MR, et al. A genome-wide association study of type 2 diabetes in Finns detects multiple susceptibility variants. Science. 2007;316:1341-5.

13. Meigs JB, Shrader P, Sullivan LM, McAteer JB, Fox CS, Dupuis J, et al. Genotype score in addition to common risk factors for prediction of type 2 diabetes. N Engl J Med 2008;359:2208-19.

14. Kahn SE, Hull RL, Utzschneider KM. Mechanisms linking obesity to insulin resistance and type 2 diabetes. Nature. 2006;444:840-6.

15. Feinberg AP. The key role of epigenetics in human disease prevention and mitigation. $\mathrm{N}$ Engl $\mathrm{J}$ Med 2018;378:1323-1334.

16. Barroso M, Florindo C, Kalwa H, Silva Z, Turanov AA, Carlson B, et al. Inhibition of cellular methyltransferases promotes endothelial cell activation by suppressing glutathione peroxidase 1 protein expression. J Biol Chem. 2014;289:1535062.

17. Sommese L, Zullo A, Mancini FP, Fabbricini R, Soricelli A, Napoli C, et al. Clinical relevance of epigenetics in the onset and management of type 2 diabetes mellitus. Epigenetics. 2017;12:401-15.

18. Sommese L, Benincasa G, Lanza M, Sorriento A, Schiano C, Lucchese R, et al. Novel epigeneticsensitive clinical challenges both in type 1 and type 2 diabetes. J Diabetes Complications. 2018;32:1076-84.

19. Keating ST, Plutzky J, El-Osta A. Epigenetic changes in diabetes and cardiovascular risk. Circ Res. 2016;118:1706-22.

20. Wei Y, Yang CR, Wei YP, Zhao ZA, Hou Y, Schatten $\mathrm{H}$, et al. Paternally induced transgenerational inheritance of susceptibility to diabetes in mammals. Proc Natl Acad Sci U S A. 2014;111:1873-8.

21. De Nigris F, Cacciatore F, Mancini FP, Vitale DF, Mansueto G, D'Armiento FP, et al. Epigenetic hallmarks of fetal early atherosclerotic lesions in humans. JAMA Cardiol. 2018;3:1184-91.

22. Napoli C, Infante T, Casamassimi A. Maternalfoetal epigenetic interactions in the beginning of cardiovascular damage. Cardiovasc Res. 2011;92:367-374.

23. Napoli C, Crudele V, Soricelli A, Al-Omran M, Vitale N, Infante $\mathrm{T}$, et al. Primary prevention of atherosclerosis: a clinical challenge for the reversal of epigenetic mechanisms? Circulation. 2012;125:2363-73.

24. Santolini M, Barabási AL. Predicting perturbation patterns from the topology of biological networks. Proc Natl Acad Sci U S A. 2018;115:6375-83.

25. Barabási AL, Gulbahce N, Loscalzo J. Network medicine: a network-based approach to human disease. Nat Rev Genet. 2011;12:56-68.

26. Barabási AL. Network medicine-from obesity to the "diseasome". N Engl J Med. 2007;357:404-7.

27. Sharma A, Halu A, Decano JL, Padi M, Liu YY, Prasad RB, et al. Controllability in an islet specific regulatory network identifies the transcriptional factor NFATC4, which regulates type 2 diabetes associated genes. NPJ Syst Biol Appl. 2018;4:25.

28. Liu H, Wang T, Liu H, Wei Y, Zhao G, Su J, et al. Detection of type 2 diabetes related modules and genes based on epigenetic networks. BMC Syst Biol. 2014;8:1-5.

29. Gong R, Chen M, Zhang C, Chen M, Haibin L. A comparison of gene expression profiles in patients with coronary artery disease, type 2 diabetes, and their coexisting conditions. Diagn Pathol. 2017;12:44.

30. Willmer T, Johnson R, Louw J, Pheiffer C. Bloodbased DNA methylation biomarkers for type 2 diabetes: potential for clinical applications. Front Endocrinol (Lausanne). 2018;9:744.

31. Selbach M, Schwanhäusser B, Thierfelder N, Fang Z, Khanin R, Rajewsky N. Widespread changes in protein synthesis induced by microRNAs. Nature. 2008;455:58-63.

Cite this article as: Benincasa G, Marfella R, Schiano C, Napoli C. Perturbation of interactome through micro-RNA and methylome analysis in diabetes endophenotypes: the PIRAMIDE pathogenic clinical study design. Int J Clin Trials 2019;6(3): 117-21. 